\title{
Comparison of remifentanil versus fentanyl general anesthesia for short outpatient urologic procedures
}

\author{
ANTHONY L. KOVAC • KARRI L. SUMMERS
}

\begin{abstract}
Study objectives. To compare the effect of remifentanil versus fentanyl isoflurane general anesthesia on Aldrete score, emergence, extubation and discharge times from the operating room (OR) and postanesthesia care unit (PACU) following short outpatient urologic procedures (panendoscopy and cystoscopy, bladder hydrodilatation, stent placement).

Patients and methods. 40 patients 18 years of age or older scheduled for short elective outpatient urological procedures with an expected duration of less than 30 minutes.

Following Institutional Review Board (IRB) approval and written informed consent, 40 American Society of Anesthesiologists (ASA) physical class 1-3 adult outpatients were enrolled and equally $(n=20)$ randomized into remifentanil and fentanyl groups. Preoperatively, all subjects received intravenous (IV) midazolam 1-2 mg and were induced with propofol $2 \mathrm{mg} /$ $\mathrm{kg}$ IV. Muscle relaxation was achieved with succinylcholine or rocuronium, followed by intubation. The remifentanil group received remifentanil $1 \mu \mathrm{g} / \mathrm{kg} \mathrm{IV}$ at induction with a maintenance dose of remifentanil 0.1 to $2 \mu \mathrm{g} / \mathrm{kg} / \mathrm{min} I \mathrm{~V}$ in the presence of $60 \%$ nitrous oxide $\left(\mathrm{N}_{2} \mathrm{O}\right) / 40 \%$ oxygen $\left(\mathrm{O}_{2}\right)$ and end-tidal isoflurane of 0.3 to $0.4 \%$ (for amnesia). The fentanyl group received fentanyl $2 \mu \mathrm{g} / \mathrm{kg} \mathrm{IV}$ at induction, maintenance dose of fentanyl 2 to $3 \mu \mathrm{g} / \mathrm{kg} \mathrm{IV}$ intermittent bolus, and 60\% $\mathrm{N}_{2} \mathrm{O} / 40 \%$ $\mathrm{O}_{2}$ with $2 \%$ end-tidal isoflurane. Muscle relaxation was reversed at the end of anesthesia as needed. Times for OR entry, emergence, extubation, total OR time (entry to exit) and PACU discharge time, as well as Aldrete scores at time of OR exit and PACU discharge were determined. Data was evaluated by ANOVA, t-test and Mann-Whitney tests. A p $<0.05$ value was considered statistically significant.

Results. There was no significant difference between groups in age, gender, weight, ASA class, PACU analgesic or antiemetic use, or times of emergence, extubation, OR exit and PACU discharge. There was a significant difference $(p<0.05)$ in OR exit Aldrete score but not PACU discharge Aldrete score. No adverse events were noted.

Conclusions. While there was no difference between the remifentanil and fentanyl groups regarding recovery time from $O R$ and PACU, remifentanil patients had significantly better OR exit Aldrete scores with less sedation upon arrival at phase I PACU recovery than the fentanyl group. This anesthesia technique may prove helpful for fast-track eligibility of these patients.
\end{abstract}

Key words: remifentanil, fentanyl, isoflurane, general anesthesia, urologic procedures, outpatient surgery, Aldrete score, recovery time, discharge time

\section{Introduction}

Recent advancements in urology procedures and instruments have resulted in an increased number of minimally invasive outpatient urological procedures. (1) These procedures, while short in duration ( $\leq 30-40$ minutes), can intraoperatively be stimulating and uncomfortable for the patient, but postoperatively may be associated with minimal pain. Patient emergence, extubation and discharge from the operating room and postanesthesia care unit are important time points for recovery of outpatients receiving general anesthesia. The goal of outpatient anesthesia and analgesia is to provide a surgical stage of anesthesia that can be induced quickly and resolved rapidly after discontinuing its administration, allowing for the rapid recovery of the patient to baseline pre- 
anesthetic mental and physical functioning. Anesthesia during urologic procedures provides for patient comfort, and by immobilizing the patient increases patient safety as perforation of the bladder or ureter resulting from patient movement can occur when these areas are instrumented. $(1,2)$ Outpatient anesthesia consists of a variety of anesthetic combinations, which include intravenous sedatives, hypnotics, and opioids as well as inhalation agents.

Remifentanil (UltivaTM; Abbott Labs, Inc., Abbott Park, IL) is an ultra-short acting opioid with a serum half-life of nine minutes. Remifentanil is metabolized by nonspecific plasma and tissue esterases, resulting in the rapid offset of effect when its administration is discontinued. $(3,4)$ Prior research has determined that remifentanil: 1 ) is effective in blunting the increased hemodynamic responses of heart rate $(\mathrm{HR})$ and blood pressure (BP) following induction of anesthesia and surgical stimuli; (5) 2) decreases the minimum alveolar concentration (MAC) requirements of inhalation agents; (6) 3) produces dose-dependent analgesia when administered as part of a balanced anesthetic technique. (7) Due to the short duration of action of remifentanil, an increase in postoperative pain may occur once its administration is discontinued. Procedures that are associated with moderate to major postoperative pain require the addition of longer acting pain medications (i.e. fentanyl, morphine) during the end of anesthesia and prior to the end of remifentanil infusion to ensure patient analgesia and comfort.

Patient emergence, extubation and discharge from the operating room and post anesthesia care unit are important time points for recovery of outpatients receiving general anesthesia. Due to its ultrashort duration of action and rapid recovery time, remifentanil appears to be an ideal drug to incorporate into a balanced $\mathrm{N}_{2} \mathrm{O}, \mathrm{O}_{2}$, opioid, muscle relaxant anesthesia plan for brief outpatient procedures associated with minimal or no postoperative pain.

Cost containment is a continual and important concern in health care. While remifentanil has a higher $\mu \mathrm{g} / \mathrm{dollar}$ cost compared to older, longer acting opioids (morphine, fentanyl) or inhalation anesthetic agents (halothane, isoforane), the time in the OR or PACU theoretically could be decreased by remifentanil use. The purpose of this study was to compare the effect of remifentanil versus fentanyl isoflurane general anesthesia on Aldrete scores, emergence, extubation and discharge times from OR and PACU following short outpatient urologic procedures (panendoscopy and cystoscopy, bladder hydrodilatation, stent placement) with an expected duration of less than 30 minutes.

\section{Methods}

Following IRB approval and written informed consent, 40 ASA physical class 1-3 adult male and female patients scheduled for outpatient urological procedures under general anesthesia were enrolled in this randomized, prospective, openlabel, single-blind study. Inclusion and exclusion criteria are listed in table 1. Subjects were randomly assigned to one of two study groups (remifentanil or fentanyl) by picking numbers from a hat. Baseline vital signs were obtained and included BP via non-invasive cuff, $\mathrm{HR}$ via electrocardiogram, and $\mathrm{O}_{2}$ saturation $\left(\mathrm{SpO}_{2}\right)$ via pulse oximeter. Study subjects were equally divided $(n=20$

\section{Table 1. Inclusion/Exclusion criteria.}

Inclusion Criteria

1. ASA physical class I, II or III

2. Females or males age 18 years or older

3. Subjects who are scheduled for outpatient urological procedures requiring general anesthesia

\section{Exclusion Criteria}

1. Subjects who were scheduled for admission to the hospital postoperatively

2. ASA physical class IV or V

3. Pregnant females

4. Subjects who were dependent on opioids

5. Subjects with allergies to opioids, eggs, soy protein or propofol

ASA, American Society of

Anesthesiologists each) into remifentanil and fentanyl groups. All subjects received midazolam 1-2 mg IV as premedication, and general anesthesia was induced with propofol $2 \mathrm{mg} / \mathrm{kg}$ IV. Muscle relaxation was accomplished with succinylcholine or rocuronium, followed by intubation. The remifentanil group received remifentanil $1 \mu \mathrm{g} / \mathrm{kg} \mathrm{IV}$ at induction and a maintenance dose of remifentanil 0.1 to $2.0 \mu \mathrm{g} / \mathrm{kg} / \mathrm{min}$ IV in the presence of $60 \% \mathrm{~N}_{2} \mathrm{O} / 40 \% \mathrm{O}_{2}$ and 0.2 to $0.4 \%$ end-tidal isoflurane (for amnesia). The fentanyl group received fentanyl $2 \mu \mathrm{g} /$ $\mathrm{kg} I V$ at induction, a maintenance dose of fentanyl 2 to $3 \mu \mathrm{g} / \mathrm{kg} \mathrm{IV}$ in the presence of $60 \% \mathrm{~N}_{2} \mathrm{O} / 40 \% \mathrm{O}_{2}$ and $2 \%$ end-tidal isoflurane. When surgery was completed, all anesthetic agents were discontinued, and study subjects were allowed to recover from anesthesia. Muscle relaxation was reversed at the end of anesthesia as needed. The endotracheal tube was removed when the subjects' respiratory rate was $\geq 12$ breaths per minute and they could lift their heads for three seconds, squeeze their hand and open their eyes on command. Study subjects were then moved to a cart and transported to the PACU with $100 \% \mathrm{O}_{2}$ via a face mask. Times for OR entry, emergence, extubation, total OR (entry to exit) and PACU discharge, as well as Aldrete scores at time of OR exit and PACU discharge were determined. Protocol schema is diagrammed in figure 1.

The time points studied were elapsed time from: 1) end of operation to extubation; 2) end of operation to exit from the OR suite; 3) OR exit to arrival at phase 1 PACU recovery: 4) phase 1 exit to discharge from phase $2 \mathrm{PACU}$ recovery. Variables also analyzed were the type and amount of opioid and antiemetic medications used in the PACU. Postoperative care for analgesics and antiemetics was conducted according to PACU protocol.

Demographic data were obtained on each patient, including age, weight, height, gender and ASA classification. Medications, dosages, and time of initiation and discontinuation of study medications were determined, inclu- 


\begin{tabular}{|c|c|c|}
\hline & \multicolumn{2}{|c|}{ Meet inclusion criteria } \\
\hline & \multicolumn{2}{|c|}{ Preoperative Midazolam 1-2 mg IV } \\
\hline & \multicolumn{2}{|c|}{ Randomization } \\
\hline & Remifentanil Group & Fentanyl Group \\
\hline & $(n=20)$ & $(n=20)$ \\
\hline \multirow{3}{*}{ Anesthesia Induction } & Propofol 1-2 mg/kg & Propofol $1-2 \mathrm{mg} / \mathrm{kg}$ \\
\hline & Remifentanil $0.5-1.0 \mu \mathrm{g} / \mathrm{kg}$ & Fentanyl $1-2 \mu \mathrm{g} / \mathrm{kg}$ \\
\hline & $\begin{array}{l}\text { Muscle relaxant for } \\
\text { Intubation }\end{array}$ & Muscle relaxant for \\
\hline \multirow{7}{*}{ Anesthesia Maintenance } & & \\
\hline & $\mathrm{N}_{2} \mathrm{O} / \mathrm{O}_{2} 60 \% / 40 \%$ & $\begin{array}{l}\mathrm{N}_{2} \mathrm{O} / \mathrm{O}_{2} 60 \% / 40 \% \\
\text { Isoflurane } 1-2 \% \text { end-tidal }\end{array}$ \\
\hline & $\begin{array}{l}\text { Isoflurane } 0.4 \% \text { end-tidal } \\
\text { Remifentanil } 0.1-2 \mu \mathrm{g} / \mathrm{kg}\end{array}$ & $\begin{array}{l}\text { Isoflurane } 1-2 \% \text { end-tidal } \\
\text { Fentanyl } 2-3 \mu \mathrm{g} / \mathrm{kg}\end{array}$ \\
\hline & Muscle relaxant prn & Muscle relaxant prn \\
\hline & $\begin{array}{l}\text { Muscle relaxant reversal } \\
\text { (as needed) }\end{array}$ & $\begin{array}{l}\text { Muscle relaxant reversal } \\
\text { (as needed) }\end{array}$ \\
\hline & Emergence & Emergence \\
\hline & Awakening & Awakening \\
\hline
\end{tabular}

Figure 1. Protocol schema.

ding the type and amount of IV fluids administered. Information regarding the type of procedure performed, operative start and stop times, OR arrival and departure times, time of placement and removal of the endotracheal tube, and details regarding any perioperative adverse events were noted. An Aldrete score was assessed for each patient at the time of departure from the OR suite and at the time PACU discharge criteria were met. A special notation was made if the subject met discharge criteria but was unable to leave the PACU for reasons beyond the control of the investigator. Postoperative information included PACU arrival and departure times and the type and amount of IV fluids or other medications (opioids, antiemetics) administered. Any adverse events were noted. All subjects were interviewed within 24 hours of hospital discharge regarding their perception of their anesthetic experience and the occurrence of any adverse events.

This study compared time elapsed from the end of the surgical procedu-

e to extubation and exit from the OR between subjects receiving remifentanil versus fentanyl general anesthesia. The degree of discharge readiness, use of opioids or antiemetics in the PACU, and the amount of time the subjects were in the PACU were compared between groups. Data were evaluated by ANOVA, t-test and Mann-Whitney tests. $A p<0.05$ value was set for statistical significance.

\section{Results}

There was no difference between groups in age, gender, weight, height, ASA physical class, PACU analgesic (fentanyl) or antiemetic use (table 2). There was no difference between groups in total OR medications (table 3). While there was no difference in times of emergence, extubation, OR exit and PACU discharge. There was a significant difference $(p<0.05)$ between groups in OR exit Aldrete score but not PACU discharge Aldrete score (table 4). No adverse events were noted. No statistically significant difference was found in the occurrence of postoperative nausea and vomiting (PONV) or postoperative pain between the two study groups. However, the PACU times for subjects who experienced both PONV and pain in both study groups was substantially longer than subjects who experienced none or only one of these postanesthesia adverse events. No statistically significant difference was found between groups for the time from the end of the procedure to extubation and departure from the OR suite. Only a marginal statistically significant difference $(p=0.058)$ was found between groups regarding the PACU length of stay (mean \pm SD) with the time being $50.1 \pm 10.87$ and $63.0 \pm$ 12.67 minutes for the remifentanil and fentanyl groups, respectively. However, there appeared to be clinical significance since the remifentanil group had attained a higher Aldrete score (10.0) earlier (at the time of OR exit) and was able to leave the PACU within 60 minutes on average of arrival.

\section{Discussion}

The progression of many urology procedures from inpatient to outpatient status has occurred over the past several years. Continued advancement in surgical equipment and techniques, as well as an increased focus on cost containment has facilitated this change in urology. (2) A retrospective study by Kaye (1) determined that urology outpatient procedures increased from $26 \%$ in 1987 to $42 \%$ in 1992 . The surgical sites with the largest increases in outpatient urology procedures included the kidney, ureter, and urethra.

With an increasing number of outpatient surgical procedures, the field of anesthesia is challenged with developing an anesthetic plan that provides patient comfort and optimal conditions for the surgeon, allowing for rapid patient emergence, recovery and discharge with minimal or no postanesthesia adverse events. This has led to the research and development of short acting anesthetic medications 
Table 2. Patient demographics.

\begin{tabular}{cccc}
\hline & REMIFENTANIL & FENTANYL & $P$ \\
\hline $\mathrm{n}$ & 20 & 20 & \\
\hline+ Age, years & $48(28-60)$ & $40(25-55)$ & 0.0791 \\
\hline *Weight, $\mathrm{kg}$ & $77.20 \pm 14.49$ & $81.00 \pm 25.88$ & 0.0956 \\
\hline Height, in & $66.25 \pm 3.9$ & $66.68 \pm 4.41$ & 0.494 \\
\hline Gender & & & \\
Males & 8 & 11 & \\
Females & 12 & 9 & \\
\hline ASA & & & \\
1 & 2 & 3 & \\
2 & 12 & 12 & \\
3 & 6 & 5 &
\end{tabular}

*Above weight and height values are mean \pm SD

+ Above age values are expressed as median (range)

ASA, American Society of Anesthesiologists.

that enable patients to regain cognitive and psychomotor functions in a more timely fashion, with minimal side effects allowing for earlier discharge home. Remifentanil has been investigated and is used as a short acting opioid in balanced anesthesia for outpatient surgery. Burkle and colleagues, (5) determined that remifentanil was an ideal opioid for short, painful procedures in the OR, emergency room and intensive care units. They emphasized that remifentanil does not provide for postoperative analgesia when its administration is discontinued. Thus, the initiation of longer acting analgesics or local anesthetic techniques prior to emerging from anesthesia is required to prevent the immediate onset of pain and discomfort after completion of procedures associated with continued postoperative pain.

Thompson et al. (8) determined that remifentanil effectively attenuated sympathetic stimulation induced by orotracheal intubation. Lang et al. (6) found that remifentanil caused a corresponding reduction in the minimum alveolar concentration of isoflurane. Both the remifentanil and fentanyl groups in our study utilized isoflurane as an inhalation agent. However, the use of isoflurane was different between groups. In the remifentanil group, a low end-tidal dose of isoflurane 0.3 to $0.4 \%$ ( $1 / 4$ to $1 / 3 \mathrm{MAC}$ ) was used as the secondary anesthetic for amnesia in combination with remifentanil as the primary anesthetic for analgesia. In the isoflurane group, a $2 \%$ end-tidal isoflurane concentration (1.67\% MAC) was used as the primary anesthetic in combination with fentanyl as the secondary anesthetic. The anesthetic end-point for both groups was the blunting of intraoperative surgical stress responses as exhibited by tachycardia and hypertension.

There continues to be an increase in the number of short, stimulating outpatient urology procedures such as panendoscopy and cystoscopy, bladder hydrodilatation for interstitial cystitis, ureteral stent placement and extracorporeal shock wave lithotripsy for kidney or ureteral stones. $(1,2)$ These procedures usually require more sedation and analgesia than can be given by monitored anesthesia care alone, as often these procedures are associated with moderate intraoperative and postoperative pain and discomfort. With an increased emphasis on cost containment and shorter PACU stays, remifentanil appeared to be an ideal opioid choice for balanced anesthesia during these short outpatient urological cases. However, the present study determined no clinically significant (mean time difference of $\approx 1.5$ minutes) or statistically significant difference between the time from the end of the surgical procedure to the time of extubation comparing remifentanil to the fentanyl.

No clinical or statistical significance was found comparing the difference in time spent in the OR suite after termination of the surgical procedure to departure from the OR suite. The fentanyl group (mean \pm SD, 9.55 \pm 2.54 minutes) exited 0.3 minutes earlier than the remifentanil group ( $9.85 \pm 4.40$ minutes, table 4$)$. Remifentanil may prove to be useful to shorten recovery time following long surgical procedures. However, compared to fentanyl, remifentanil did not shorten OR exit time following short 30-40 minute urological procedures.

Comparison of the difference in the amount of time each group spent in the PACU revealed only marginal statistical significance $(p=0.058)$. This marginal difference in PACU time may be attributed to the more rapid attainment of discharge criteria to an Aldrete score of 10 by the remifentanil group. However, there was no significant difference between groups in PACU Aldrete discharge scores at the time of PACU discharge. While there was no difference between groups regarding recovery times from OR and PACU, the remifentanil groups had significantly better OR exit Aldrete scores (10 out of 10) with less sedation than the fentanyl group (9 out of 10). The subjects in the remifentanil group had mean $( \pm S D)$ Aldrete scores of 9.50 \pm 0.51 upon discharge from the OR suite as opposed to a mean score of $8.65 \pm 1.34$ for subjects in the fentanyl group. This finding may be attributed to remifentanil's short elimination half-life and less sedation in conjunction with a lower end-tidal isoflurane concentration compared to the fentanyl-isoforane group. This could have clinical and economic significance, since the remifentanil group achieves an Aldrete score of 10 earlier than the fentanyl group, and on average was able to leave the PACU within 60 minutes of arrival.

There was no statistical difference in the total PACU use (mean \pm SD) of opioids (fentanyl) $(50 \pm 9.8 \mu \mathrm{g})$ versus remifentanil $(60.71 \pm 10.2 \mu \mathrm{g})$ between the two groups. The fentanyl group included study subjects who were successfully treated with IV ketorolac (30 mg) and acetaminophen $(650 \mathrm{mg}$ ) as opposed to only opioid medications. It should be noted that the differences of anal- 
gesic choice between the remifentanil and fentanyl groups were related more to the first-line opioid chosen by the anesthesia provider as opposed to the severity of the pain experienced by the study subjects.

The number of patients experiencing postoperative pain in the PACU was identical $(n=6)$ in the two study groups. The mean visual analog pain score reported for those experiencing postoperative pain in the fentanyl group was 4.8 compared to a score of 5.4 in the remifentanil group (visual analog scale from 0 , no pain, to 10 , maximum pain). The remifentanil group had two outlying pain scores of 10 . One subject was a 25-year old female who required 300 $\mu \mathrm{g}$ IV fentanyl, $30 \mathrm{mg}$ IV ketorolac and two oxycodone / acetaminophen oral pain medications to relieve her postoperative pain. Fifty-five minutes were required to attain adequate pain control for this subject, which resulted in a total PACU time of 86 minutes. The other subject who reported a pain score of 10 was a 37 -year-old female who preoperatively reported having chronic pain (equal to a pain score of 6 to 7 out of a possible 10) on a daily basis. This subject was successfully treated with two oxycodone / acetaminophen tablets and one B \& O (Belladonna and Opium) suppository. Total PACU time for this subject was 65 minutes, which was approximately 15 minutes longer than the mean PACU time for this study group. If the aforementioned outlying patient who required $300 \mu \mathrm{g}$ of fentanyl is excluded, the mean $( \pm S D)$ dose of PACU fentanyl becomes $41.67 \pm 11.6$ $\mu \mathrm{g}$ for the fentanyl group compared to $60.71 \pm 10.2 \mu \mathrm{g} / \mathrm{kg}$ for the remifentanil group. This does not appear to have any clinical or cost significance, as the doses of IV opioids are administered from ampoules that contain either 100 or $500 \mu \mathrm{g}$ of fentanyl. In our hospital pharmacy, excess IV opioid medications cannot be used for other patients. Any unused portion is subsequently wasted, with the entire ampoule being charged to that patient.

The incidence of PONV was similar, occurring in 3 and 4 patients for the

Table 3. Operating room medications, hypnotics, IV fluids, opioids, antiemetics.

\begin{tabular}{lccc}
\hline & Remifentanil & Fentanyl & $p$ \\
\hline $\begin{array}{l}\text { Total Propofol } \\
\text { induction } \\
\text { dose(mg) }\end{array}$ & 20 & 20 & \\
\hline $\begin{array}{l}\text { Total Midazolam } \\
\text { preop dose }(\mathrm{mg})\end{array}$ & $141.00 \pm 34.47$ & $177.00 \pm 31.61$ & 0.0769 \\
\hline $\begin{array}{l}\text { Total patients } \\
\text { PACU analgesic }\end{array}$ & $7(35 \%)$ & $1.87 \pm 0.32$ & 0.0941 \\
$\begin{array}{l}\text { use } \mathrm{n}(\%) \\
\text { Total patients }\end{array}$ & $3(15 \%)$ & 0.144 \\
$\begin{array}{l}\text { PACU antiemetic } \\
\text { use } \\
\mathrm{n}(\%)\end{array}$ & & & \\
\hline $\begin{array}{l}\text { Total PACU } \\
\text { fentanyl use }(\mu \mathrm{g})\end{array}$ & $60.71 \pm 10.2$ & $50.0 \pm 9.8$ & 0.0376 \\
\hline
\end{tabular}

PACU, postanesthesia care unit.

fentanyl and remifentanil groups, respectively. The mean nausea scores ( $1=$ no nausea, $10=$ worst nausea $)$ were 1 and 2, for the fentanyl and the remifentanil groups, respectively. The number of antiemetic doses required to treat PONV in the PACU was 4 and 2 doses, respectively, for the fentanyl and remifentanil groups (table 3 ).

The PACU times associated with patients who experienced postoperative complications such as pain, nausea and vomiting were substantially longer than patients who had no postoperative complications. The mean $( \pm$ SD) PACU time for all subjects in the study who had an uneventful recovery was $51.36( \pm 15.56)$ minutes. Interestingly, three subjects experienced only PONV and had a mean PACU time of 42.33 ( \pm 13.42$)$ minutes. Eight subjects experienced only pain and had a mean PACU time of 59.145 ( \pm 20.52 ) minutes. Four subjects experienced both PONV and pain and had a mean PACU time of $94.5( \pm 22.4)$ minutes. These findings continue to support previous literature findings regarding increased length of stay and costs associated with these postoperative complications. $(9,10)$ These subjects had increased postoperative costs due to increased recovery time and additional medication and supply charges.
Propofol was used as the IV induction agent in this study. Numerous studies $(7,11,12)$ have compared propofol and thiopental for induction of anesthesia in the outpatient population and have determined that recovery from propofol occurs significantly more rapidly than from thiopental. Korttila et al. (12) found impaired objective performance on psychomotor tests at one hour after propofol with no detectable impairment after three hours. However, subjects who received thiopental had significantly impaired psychomotor performance at five hours after administration. An additional advantage of propofol is its antiemetic effects. $(7,11)$

Studies conducted by Sengupta and Plantevin (13) and Sukhhani et al. (14) determined that the addition of $\mathrm{N}_{2} \mathrm{O}$ to propofol for maintenance of anesthesia in outpatient laparoscopic procedures reduced the amount of propofol required to maintain anesthesia. This resulted in a significantly shorter time to eye opening and orientation than in patients who did not receive $\mathrm{N}_{2} \mathrm{O}$.

Remifentanil is an ultra-short acting opioid that produces effects similar to other opioids, including attenuation of hemodynamic responses to noxious stimuli and dose-dependent reductions in the MAC of volatile anesthetics. $(4,8)$ The decreased dose of 0.3 to $0.4 \%$ ( $1 / 4$ to $1 / 3$ 
Table 4. Study variables.

\begin{tabular}{llll}
\hline & & & \\
& Remifentanil & Fentanyl & $p$ \\
\hline Aldrete & $9.50 \pm 0.51^{*}$ & $8.65 \pm 1.34$ & 0.0493 \\
OR exit & $10.0 \pm 0.0$ & $10.0 \pm 0.0$ & \\
Scores & & & \\
PACU discharge & & & 0.0812 \\
\hline PACU Time (min) & $50.1+10.87$ & $63.0 \pm 12.67$ & \\
& & & \\
\hline
\end{tabular}

${ }^{*} \mathrm{p}<0.05$ compared to fentanyl group

Data values $=$ mean \pm SD

PACU, postanesthesia care unit; OR, operating room.

MAC) of isoflorane was used in the remifentanil groups to help ensure amnesia. Remifentanil, like other opioids, has been shown to reduce the MAC of volatile anesthetic agents in a dose-dependent fashion. Burke et al. (5) determined that the MAC of isoflurane was decreased by 12 to $91 \%$ depending on the plasma concentration of remifentanil. Lange and coworkers (6) have suggested that, due to its short duration of action, remifentanil should be maintained at higher levels initially and titrated to clinical effect while administering an anesthetic gas, such as isoflurane, at levels slightly above MAC awake. They suggested that this will maintain an adequate anesthetic state, allowing for rapid awakening without a prolonged recovery time due to respiratory depression.

The chemical structure of remifentanil is metabolized by nonspecific blood and tissue esterases with no dependence on redistribution or liver metabolism, resulting in its rapid and immediate elimination upon discontinuation. Remi- fentanil has an elimination half-life of approximately 9 minutes. $(4,5)$ Prolonged administration or continuous infusion of remifentanil does not result in tissue accumulation or alter its half-life and speed of elimination. $(3,4)$ Remifentanil does not provide postoperative analgesia when its administration is discontinued. Thus, prior to emergence from anesthesia, it is necessary to initiate longer acting analgesics, local anesthetics or other means of pain control to prevent the immediate onset of pain after the completion of surgical procedures associated with moderate to severe postoperative pain.

With the continued increase in the number of short, stimulating urological procedures that are associated with minimal postoperative discomfort, and the increased focus on cost containment with shorter PACU stays, remifentanil appears to be an alternative opioid choice for balanced anesthesia in these cases, allowing for rapid recovery and the "fast tracking" of these outpatients. However, except for less sedation at the time of OR exit, remifentanil offered no additional advantages over the standard fentanyl isoforane general anesthesia technique for these short outpatient urology procedures. An explanation for this could be the relatively short duration of the urological procedures chosen, $34.45 \pm 22.10$ (mean \pm SD) minutes, for the fentanyl group compared to $41.85 \pm 20.21$ for the remifentanil group, with no statistical difference in duration between the two groups. For short, low-stimulating surgeries, the difference in \% end tidal isoflurane concentration (maximum \% minus minimum \%) used for inhalation anesthesia between the remifentanil and fentanyl groups 1.33\% (1.63 minus $0.31 .7 \%$ ) and (2.0 minus $0.3 \%)$, respectively, was small. In addition, in relatively lean patients during short surgical procedures, the amount of isoflurane gas absorbed by the study patients was not large enough to prolong their wake-up. In conclusion, with an increased number of outpatient urological surgery procedures, there is a challenge to provide analgesia and amnesia while allowing for rapid recovery and minimal postanesthesia complications. For short outpatient urologic procedures, there was no difference between remifentanil and fentanyl isoflurane general anesthesia regarding anesthesia duration, extubation, OR exit and PACU discharge times. However, remifentanil patients had significantly better OR exit Aldrete scores with less sedation than the fentanyl group. This may prove helpful for the fast-track eligibility of these patients and deserves further investigation.

\section{REFERENCES}

1. Kaye KW. Changing trends in urology practice: increasing outpatient surgery. Austr New Zealand J Surg 1995;65(1):31-4

2. Kahn RL. Outpatient endourlogic procedures. Urologic Clin North Amer 1987;14(1):77-89.

3. Westmoreland CL, Hoke JF, Sebel PS, Hug CC Jr, Muir KT. Pharmacokinetics of remifentanil (Gl87084B) and its major metabolite (GI90291) in patients undergoing elective inpatient surgery. Anesthesiology 1993;79:893-903.

4. Glass PS, Hardman D, Kamiyama Y, Quill TJ, Marton G, Donn KH, et al. Preliminary pharmacokinetics and pharmacodynamics of an ultra-short-acting opioid: L remifentanil (GI87084B). Anesth Analg 1993;77(5):1031-40

5. Burkle H, Dunbar S, Van Aken H. Remifentanil: a novel, short-acting, mu-opioid. Anesth Analg 1996;83(3):646-51. 
6. Lange E, Kapila A, Shlugman D, Hoke JF, Sebel PS, Glass PSA. Reduction of isoflurane minimal alveolar concentration by remifentanil. Anesthesiology 1996;85(4):721-8.

7. Gupta A, Larsen LE, Sjoberg F, Lindh ML, Lennmarken C. Thiopentone or propofol for induction of isoflurane-based anesthesia for ambulatory surgery? Acta Anaesthesiol Scand 1992;36:670-4.

8. Thompson JP, Hall AP, Russell J, Cagney B, Rowbotham DJ. Effect of remifentanil on the hemodynamic response to orotracheal intubation. Br J Anaesth 1998;80(4):467-9.

9. Hirsch J. Impact of postoperative nausea and vomiting in the surgical setting. Anesthesia 1994;49:30-3.

10. Carroll NV, Miederhoff PA, Cox FM, Hirsch JD. Costs incurred by outpatient surgical centers in managing nausea and vomiting. J Clin Anesth 1994;6(5):364-9.

11. Johnston R, Noseworthy T, Anderson B, Konopad E, Grace M. Propofol versus thiopental for outpatient anesthesia. Anesthesiology 1987;67(3):431-3.

12. Korttila K, Nuotto EJ, Lichtor JL, Ostman PL, Apfelbaum J, Rupani G. Clinical recovery and psychomotor function after brief anesthesia with propofol or thiopental. Anesthesiology 1992;76:676-81.

13. Sengupta P, Plantevin OM. Nitrous oxide and day-case laparoscopy: effects on nausea, vomiting and return to normal activity. Br J Anaesth 1988;60:570-3

14. Sukhani R, Lurie J, Jabamoni R. Propofol for ambulatory gynecologic laparoscopy: does omission of nitrous oxide alter postoperative emetic sequel and recovery? Anesth Analg 1994;78(4):831-5. 